\title{
Gestión administrativa en MIPYMES sur Estado de México, 2016
}

Administrative management in MSMES southern state of Mexico, 2016

PME gestão administrativa no sul do Estado do México de 2016

DOI: http://dx.doi.org/10.23913/ricea.v6i11.87

Josué Ociel Márquez Gómez

Unidad Académica Profesional Tejupilco, Universidad Autónoma del Estado de México josuemar12@gmail.com

Daniel Cardoso Jiménez

Unidad Académica Profesional Tejupilco, Universidad Autónoma del Estado de México dcj_400@hotmail.com

Leticia Carreño Saucedo

Unidad Académica Profesional Tejupilco, Universidad Autónoma del Estado de México psicoletty@hotmail.com

Salvador Bobadilla Beltrán Unidad Académica Profesional Tejupilco, Universidad Autónoma del Estado de México sbb73@hotmail.com

\section{Resumen}

El presente estudio tiene como objetivo determinar la gestión administrativa en micros, pequeñas y medianas empresas (MIPYMES) que se dedican al comercio mayorista y minorista en el Sur del Estado de México, específicamente en los municipios de Amatepec, Luvianos, San Simón de Guerrero, Tejupilco, Tlatlaya, Temascaltepec y Sultepec, durante 2016. El criterio de elección de la muestra fue por tabla de números aleatorios en 332 empresas. La investigación fue descriptiva transeccional y no experimental, para lo cual se diseñó un instrumento a partir de los indicadores de operatividad de las variables a investigar; la medición se hizo mediante el método de evaluación sumaria con una escala de Likert. 
Los resultados muestran que $60 \%$ de las empresas encuestadas no tiene un organigrama, lo que limita su gestión administrativa, además 50 \% de los empresarios mencionó que el número de empleados afecta la organización de la misma, $50 \%$ delega responsabilidades y $30 \%$ tiene cultura organizacional, asimismo $25 \%$ mencionó que la productividad del capital humano tiene mucho que ver con una actitud positiva, $35 \%$ dijo que se deben utilizar diversos niveles de comunicación para que haya organización en la empresa y, por último, $80 \%$ señaló que el personal realiza sus funciones de acuerdo al salario que percibe.

La gestión administrativa ayuda a las empresas a manejar una cultura organizacional que conlleve la estructuración de un organigrama, la delimitación de funciones o responsabilidades del personal, y la implementación de manuales de organización, procedimientos o software administrativos con el fin de mejorar los procesos, reducir los costos y aumentar las utilidades de la empresa.

Palabras clave: gestión administrativa, MIPYMES, capital humano.

\section{Abstract}

The present study aims to determine the administrative management in micro, small and medium-sized enterprises (MSMES) engaged in the wholesale and retail trade in the South of the State of Mexico, specifically in the municipalities of Amatepec Luvianos, San Simón de Guerrero, Tejupilco, Tlatlaya, Temascaltepec, Sultepec, during 2016. The choice of the sample was by table of random numbers in 332 companies. The research was descriptive transactional and non-experimental, for this was designed an instrument from the indicators of operation of variables to investigate; measurement was made with the summary evaluation method with a Likert scale.

The results show that $60 \%$ of the surveyed companies does not have a flow chart, which limits its administrative management, moreover $50 \%$ of employers mentioned that the number of employees affected the Organization of it, 50\% delegate responsibilities and 30\% has organizational culture, also $25 \%$ mentioned that the productivity of human capital has much to do with a positive attitude, $35 \%$ said that different levels of communication should be used to make organization in the company and, finally, $80 \%$ indicated that staff performs its functions according to the perceived salary. 
Administrative management helps companies manage an organizational culture that carries the structure of an organization chart, the delimitation of functions or responsibilities of staff, and implementation of manuals of organization, procedures or administrative software in order to improve processes, reduce costs and increase the profits of the company.

Key words: administrative management, MSMES, human capital.

\section{Resumo}

Este estudo visa determinar a gestão administrativa em micro, pequenas e médias empresas (MPMEs) envolvidos no comércio por grosso ea retalho no sul do estado do México, especificamente nos municípios de Amatepec, Luvianos, San Simon de Guerrero, Tejupilco, Tlatlaya, Temascaltepec e Sultepec durante 2016. o critério de escolha da amostra foi por tabela de números aleatórios 332 empresas. pesquisa Transeccional foi descritivo, não experimental, para o qual um instrumento foi concebido a partir dos indicadores operacionais das variáveis para investigar; A medição foi feita pelo método de avaliação de síntese com uma escala do tipo Likert.

Os resultados mostram que $60 \%$ das empresas pesquisadas não têm um gráfico, o que limita a sua gestão administrativa, e 50\% dos empregadores disseram que o número de funcionários afeta a organização do mesmo, 50\% responsabilidades delegados e 30\% têm cultura organizacional, também 25\% mencionaram que a produtividade do capital humano tem muito a ver com uma atitude positiva, 35\% disseram que eles devem usar diferentes níveis de comunicação para que haja organização na empresa e, finalmente, $80 \%$ disseram funcionários desempenha as suas funções de acordo com o salário recebido.

gestão administrativa ajuda as empresas a gerenciar uma cultura organizacional que envolve a estruturação de uma organização, a delimitação dos papéis e responsabilidades do pessoal, e implementação de manuais organizacionais, procedimentos ou software administrativo, a fim de melhorar os processos, reduzir custos e aumentar os lucros da empresa.

Palavras-chave: administração, as MPMEs, capital humano. 


\section{Introducción}

La aportación de las MIPYMES al Producto Interno Bruto (PIB) y generación de empleos del país, muestra la importancia de llevar a cabo la investigación de estas empresas. En México existen alrededor de 4 millones 15 mil unidades empresariales, de las cuales $99.8 \%$ son MIPYMES que generan $52 \%$ del PIB y $72 \%$ del empleo en el país (Pro México, 2015).

Estas empresas forman un eslabón importante en el desarrollo nacional, por lo que es necesario atender su estructura y organización para facilitar su periodo de vida e impulsarlas para que exista una adecuada competitividad en los mercados nacional y transnacional.

La competitividad hoy en día requiere de respuestas anticipadas a los retos del entorno; además, para que las MIPYMES mejoren es necesario adecuar sus estrategias, estructura organizativa y el panorama económico actual. Sin embargo, existen empresas donde persiste una actitud conservadora a la espera de introducir cambios estructurales (Elizondo, Ríos, Cabrero, Morejón, Ramos, 2011).

Existe gran diversidad de criterios para definir y clasificar a las empresas, de acuerdo a sus necesidades u objetivos particulares (Saavedra y Hernández, 2008). Es decir, cada país, a partir de su entorno y otros factores, puede adoptar determinadas clasificaciones. De acuerdo con Garza (2000), Tunal (2003), y Zevallos (2003), las variables a partir de las cuales se puede clasificar a las empresas son: número de trabajadores, tipo de producto, tamaño del mercado, inversión en bienes de producción por persona ocupada, volumen de producción o de ventas, valor de producción o de ventas, trabajo personal de socios o directores, separación de funciones básicas de producción, personales, financieras y de ventas dentro de la empresa, ubicación o localización, nivel de tecnología de producción, orientación de mercados, valor del capital invertido y consumo de energía.

En México también existe una clasificación de las empresas, como se muestra a continuación. 
Tabla 1. Clasificación de las empresas mexicanas

\begin{tabular}{|l|l|l|l|l|}
\hline Tamaño & Sector & $\begin{array}{l}\text { Rango del número } \\
\text { de trabajadores }\end{array}$ & $\begin{array}{l}\text { Rango del monto de ventas } \\
\text { anuales }(\mathrm{MDP})\end{array}$ & $\begin{array}{l}\text { Tope máximo } \\
\text { combinado } \\
(\mathrm{MDP})\end{array}$ \\
\hline Micro & Todas & Hasta 10 & Hasta $\$ 4$ & $\$ 4.60$ \\
\hline \multirow{2}{*}{ Pequeña } & Comercio & Desde 11 hasta 30 & Desde $\$ 4.01$ hasta $\$ 100$ & $\$ 93.00$ \\
\cline { 2 - 6 } & Industria y Servicios & Desde 11 hasta 50 & Desde $\$ 4.01$ hasta $\$ 100$ & $\$ 95.00$ \\
\hline \multirow{3}{*}{ Mediana } & Comercio & Desde 31 hasta 100 & Desde $\$ 100.01$ hasta $\$ 250$ & $\$ 235.00$ \\
\cline { 2 - 6 } & Servicios & Desde 51 hasta 100 & Desde $\$ 100.01$ hasta $\$ 250$ & $\$ 235.00$ \\
\cline { 2 - 6 } & Industria & Desde 51 hasta 250 & Desde $\$ 100.01$ hasta $\$ 250$ & $\$ 250.00$ \\
\hline
\end{tabular}

Fuente: Diario Oficial de la Federación, 2009.

El concepto de administración está integrado por las funciones: planear, organizar, dirigir, coordinar y controlar, que también son principios universales aplicables a cualquier tipo de organización o empresa. Existe una proporcionalidad de la función administrativa, la cual se reparte en todos los niveles de la empresa; la práctica administrativa es descrita como algo distinto de la contabilidad, las finanzas, la producción, la distribución y otras funciones típicas de los negocios (Chiavenato, 2006).

También existen dos tipos de organizaciones cuya definición depende de sus condiciones de competitividad. Las menos competitivas son las que compensan la falta de productividad con salarios bajos y, por lo tanto, no conservan un buen capital humano. Las empresas más competitivas son aquellas que cuentan con mayor productividad y retención de mejor capital humano debido a sus salarios más altos (Unger, Flores, Ibarra, 2014).

Las empresas que se preocupan por cuidar y actualizar a su personal se mantienen al margen para invertir y retener lo que necesitan. Por ello es importante observar la manera como el capital humano influye en las MIPYMES, su forma de contribuir al desarrollo para permanecer en el mercado, generar nuevas oportunidades y mejorar las estrategias que hoy en día son necesarias debido a los constantes cambios en el mundo empresarial. 
El recurso más importante de una organización es el recurso humano, puesto que es el único que puede administrar al resto de los recursos. En una empresa se debe procurar tener el personal adecuado con los conocimientos necesarios, para aprovechar al máximo el manejo de dichos recursos y conseguir así eficacia productiva (Garibaldi, Rivera, 2015).

Es importante contar con un buen sistema de control interno en las empresas, debido a que con este instrumento es más factible comprobar la eficiencia y la productividad (Apúparo, Castillo, 2012). Las micro, pequeñas y medianas empresa en México (MIPYME) son de vital importancia ya que representan $99.8 \%$ del total de las empresas, generan $71 \%$ del empleo y aportan $52 \%$ del producto interno bruto (PIB) (INEGI, 2015).

En la presente investigación se describe la metodología utilizada, cuyos resultados presentan la percepción de 332 MIPYMES del sur del Estado de México sobre la manera como llevan el proceso administrativo para el correcto funcionamiento de sus operaciones.

\section{Objetivo}

Determinar la gestión administrativa en micro, pequeñas y medianas empresas (MIPYMES) que se dedican al comercio mayorista y minorista del sur del Estado de México, en los municipios de Amatepec, Luvianos, San Simón de Guerrero, Tejupilco, Tlatlaya, Temascaltepec y Sultepec, durante 2016.

\section{Tipo de estudio}

El nivel de conocimiento científico que busca el investigador debe determinar el tipo de estudio de acuerdo al tipo de información que espera obtener, así como el nivel de análisis a realizar (Tapia, 2000).

De acuerdo a la metodología de Hernández, Fernández y Baptista (2003), existen estudios exploratorios, descriptivos, correlacionales y explicativos. Los estudios descriptivos miden de manera independiente los conceptos o variables a los que se refieren y se centran en medir con la mayor precisión posible.

La investigación fue descriptiva transeccional y no experimental. De acuerdo a la información del Directorio Estadístico Nacional de Unidades Económicas (DENUE) se puede apreciar que en el sur del 
Estado de México se encontraron 2401 MIPYMES que se dedican al comercio mayorista y minorista. http://www.beta.inegi.org.mx/app/mapa/denue/. Consultado 21/11/2016 a las 14:00 hrs.

Como instrumento para obtener los datos se aplicó una encuesta a 332 MIPYMES de manera directa, ya sea al gerente, dueño o encargado en ese momento, principalmente en el sector terciario o comercio al sur del Estado de México. El criterio de elección de la muestra fue por el tablero aleatorio de números.

Las preguntas de investigación formuladas fueron: ¿en qué grado utilizan las MIPYMES del sur del Estado de México la gestión administrativa?, ¿cuál es la problemática que tienen las MIPYMES en la implementación de la gestión administrativa?, ¿cuál es la forma en que interviene el capital humano para una gestión administrativa? Para llevar a cabo este estudio se diseñó un instrumento a partir de los indicadores de la operatividad de las variables a investigar. La medición se hizo con ayuda de un método de evaluación sumaria mediante una escala de Likert. Para la validación y estandarización del mismo instrumento se realizaron pruebas piloto con tres diferentes dueños de empresas del sur del Estado de México, tomando como base las observaciones realizadas por Likert acerca de la elección de afirmaciones adecuadas (tabla 2). 
Tabla 2. Operatividad de las variables

\begin{tabular}{|c|c|c|}
\hline $\begin{array}{l}\text { Campo de } \\
\text { estudio }\end{array}$ & Definición operacional & Indicadores \\
\hline $\begin{array}{l}\text { Gestión } \\
\text { administrativa }\end{array}$ & $\begin{array}{l}\text { Si la empresa lleva a cabo la Gestión } \\
\text { administrativa }\end{array}$ & $\begin{array}{l}\text { Organigrama de la empresa } \\
\text { Manual de organización } \\
\text { Manual de procedimientos } \\
\text { Proceso administrativo } \\
\text { Estructura organizacional } \\
\text { Apoyo de consultores }\end{array}$ \\
\hline MIPYMES & $\begin{array}{l}\text { Si afecta la organización el tamaño de la } \\
\text { empresa } \\
\text { Si el sector al que pertenecen las empresas } \\
\text { afecta las MIPYMES }\end{array}$ & $\begin{array}{l}\text { Número de empleados } \\
\text { Las ventas influyen en la } \\
\text { organización de la empresa }\end{array}$ \\
\hline \multirow{7}{*}{ Capital humano } & $\begin{array}{l}\text { Si las MIPYMES conocen el proceso para la } \\
\text { implementación del control administrativo }\end{array}$ & $\begin{array}{l}\text { Duplicidad de funciones } \\
\text { Delegación de responsabilidad } \\
\text { Cultura organizacional }\end{array}$ \\
\hline & $\begin{array}{l}\text { Si el número de los trabajadores afecta a la } \\
\text { organización de las MIPYMES }\end{array}$ & Actitud positiva, productividad \\
\hline & & Cuenta con una estructura definida \\
\hline & $\begin{array}{l}\mathrm{Si} \text { las personas identifican los niveles de } \\
\text { comunicación }\end{array}$ & $\begin{array}{l}\text { Niveles de comunicación } \\
\text { Áreas de responsabilidad }\end{array}$ \\
\hline & & $\begin{array}{l}\text { Roles que desempeña cada } \\
\text { empleado }\end{array}$ \\
\hline & & Programas de capacitación \\
\hline & & Motivación \\
\hline
\end{tabular}


Ambiente laboral

Los salarios que se perciben en la empresa

Fuente: elaboración propia

\section{Resultados}

De acuerdo a la información obtenida en las encuestas aplicadas a los 332 gerentes, encargados o dueños de los negocios, principalmente en el sector terciario o de comercio al sur del Estado de México sobre los beneficios que tienen con la aplicación de la gestión administrativa, 60 \% no cuenta con un organigrama de la empresa, $45 \%$ no conoce el manual de organización, $40 \%$ no utiliza el manual de procedimientos, $20 \%$ conoce el proceso administrativo, $20 \%$ sabe cómo está la estructura de la misma y, por último, $15 \%$ cuenta con el apoyo de consultores.

Tabla 3. Gestión administrativa, porcentajes obtenidos en la encuesta aplicada a 332 MIPYMES de los diversos municipios del sur del Estado de México.

\begin{tabular}{l|l|l}
\hline Campo de estudio & Indicadores & Resultado \\
\hline & Organigrama de la empresa & $60 \%$ \\
& Manual de organización & $45 \%$ \\
& Manual de procedimientos & $40 \%$ \\
Gestión administrativa & Proceso administrativo & $20 \%$ \\
& Estructura organizacional & $20 \%$ \\
& Apoyo de consultores & $15 \%$ \\
\hline
\end{tabular}

Fuente: elaboración propia. 
Tabla 4. MIPYMES

\begin{tabular}{l|l|l}
\hline Campo de estudio & Indicadores & Resultado \\
\hline \multirow{2}{*}{ MIPYMES } & Número de empleados & $50 \%$ \\
& Las ventas influyen en la organización de la empresa & $30 \%$ \\
& Duplicidad de funciones & $40 \%$ \\
& Delegación de responsabilidad & $50 \%$ \\
& Cultura organizacional & $30 \%$ \\
\hline
\end{tabular}

Fuente: elaboración propia.

Para $50 \%$ de los empresarios, el número de empleados afecta a la organización, $30 \%$ dice que las ventas también influyen, $40 \%$ piensa que al momento que los empleados realizan sus actividades existe duplicidad de funciones, $50 \%$ delega responsabilidades $\mathrm{y}$, por último, $30 \%$ conoce la cultura organizacional de la empresa ya que son importantes su misión, visión, objetivos y valores para determinar su rumbo.

Tabla 5. Capital Humano

\begin{tabular}{l|l|l}
\hline Campo de estudio & Indicadores & \multicolumn{1}{c}{ Resultado } \\
\hline & Actitud positiva, productividad & $25 \%$ \\
& Cuenta con una estructura definida & $30 \%$ \\
Capital Humano & Niveles de comunicación & $35 \%$ \\
& Áreas de responsabilidad & $30 \%$ \\
Roles que desempeña cada empleado & $30 \%$ \\
& Programas de capacitación & $30 \%$ \\
& Motivación & $25 \%$ \\
& Ambiente laboral & $25 \%$ \\
& Los salarios que se perciben en la empresa & $80 \%$ \\
\hline
\end{tabular}

Fuente: elaboración propia. 
Para $25 \%$ de los empresarios, una actitud positiva tiene mucho que ver con la productividad de la empresa, $30 \%$ dijo que cuenta con una estructura definida que les permite llevar a cabo sus funciones, $35 \%$ mencionó que para que haya organización se deben utilizar diversos niveles de comunicación, 30 $\%$ tiene conocimiento de su área de responsabilidad, $30 \%$ identifica la importancia de que se lleven a cabo los distintos roles a desempeñar por cada empleado, $30 \%$ aplica los diferentes programas de capacitación para obtener una mejor organización, $25 \%$ dice que la motivación ayuda a obtener un mejor rendimiento, y por último, $80 \%$ dijo que el salario que percibe el personal influye en la realización de las funciones que le corresponden.

\section{Discusión}

Los estudios realizados son muy similares a los que llevó a cabo la Encuesta Nacional sobre Productividad y Competitividad de las micro, pequeñas y medianas empresas (ENAPROCE, 2015). De las más de 4 millones de empresas existentes en México durante 2014, $97.6 \%$ son microempresas y concentran a $75.4 \%$ del personal total ocupado, seguidas de las empresas pequeñas con $2.0 \%$ y $13.5 \%$ y de las medianas que representan al $0.4 \%$ y $11.1 \%$ respectivamente. Del total de empresas consideradas, 12.6 \% imparte capacitación a su personal. Por tamaño resalta la participación de las empresas medianas y pequeñas, las cuales registran $73.7 \%$ y $55.8 \%$ del total de unidades de esos segmentos respectivamente. En las microempresas, $11.5 \%$ capacita a su personal.

El presente estudio evidencia que los procesos administrativos en las 332 MIPYMES del sur del Estado de México, que comprende los municipios de Amatepec, Luvianos, San Simón de Guerrero, Tejupilco, Tlatlaya, Temascaltepec y Sultepec, son inadecuados. El problema radica en el débil control en la planeación, organización y dirección debido a la falta de organigrama, conocimiento del proceso administrativo, cultura organizacional, comunicación y trabajo en equipo.

Para Monchon (2014), la administración consiste en coordinar y supervisar las actividades de las organizaciones para lograr que alcancen su objetivo de manera eficaz y eficiente. Las funciones básicas de la administración son: planear, organizar, dirigir y controlar.

Es indispensable que exista un proceso administrativo adecuado ya que está ligado a cada una de las áreas de la empresa, así como a las actividades de la misma, siempre en busca de los objetivos planteados. Esto traerá consigo una mejora en los procesos, reducción de costos, mayor productividad y, 
por tanto, más utilidades, las cuales se pueden invertir en infraestructura, equipo y capacitación del personal.

Según Garibaldi y Rivera (2015), el recurso más importante de una organización es el recurso humano, el único que puede administrar al resto de los recursos.

El talento humano eficiente trae grandes beneficios a las empresas, sin embargo, es un aspecto descuidado por las MIPYMES en estudio debido al desconocimiento de las funciones a desempeñar ocasionado por la alta rotación de personal, lo que a su vez afecta la comunicación y el desempeño laboral. Es importante que las personas que laboran en la empresa tengan una actitud positiva con deseo de superación profesional, lo cual conlleva el desarrollo adecuado de sus funciones dentro de la organización y, asimismo, el incremento de la productividad en la empresa.

Para Amaya (2008), cuando se tiene bien estructurado el organigrama se procede a redactar un manual de funciones que se encarga de explicar y plasmar las funciones y procesos a realizar por cada colaborador de acuerdo a su área o puesto de trabajo.

La mayoría de las MIPYMES estudiadas carecen de manuales porque no les dan la importancia que se merecen, además de que son pocas las que solicitan apoyo a consultores externos al considerarlo un gasto innecesario ya que no contemplan sus beneficios a largo plazo. La planeación es muy importante en la administración de empresas pues es un proceso de toma de decisiones que busca alcanzar los objetivos planteados tomando en cuenta la situación actual del entorno, sin embargo, los resultados demuestran que este elemento es uno de los menos trabajados debido a la falta de comunicación y al débil control, lo que genera estrategias deficientes para el logro de ventajas competitivas.

Según Garzón (2012), planificar es prever y decidir hoy las acciones que pueden llevarnos del presente al futuro deseable.

La comunicación ayuda a lograr un ejercicio eficiente del liderazgo y, en consecuencia, al cambio cultural en la organización. La comunicación es un elemento esencial en la vida de las organizaciones, su buen funcionamiento y creación de identidad. 
Una de las limitaciones de este estudio fue que los resultados no pueden generalizarse en todas las MIPYMES del sector comercio del sur del Estado de México debido a que se tomaron en cuenta solamente 332. Por ello es necesario hacer un análisis con muestras representativas de la población, ampliar el estudio para hacerlo más detallado y analizar los componentes de los factores personales del empresario que inciden en un mayor crecimiento, abordar las nuevas herramientas de la gestión empresarial que son conocidas en el resto del mundo pero desconocidas para las empresas de la región, ayudar a que se diseñen programas enfocados a desarrollar las habilidades directivas de los empresarios considerándolo un detonante positivo que impulsa la permanencia de las empresas y, por último, llevar a cabo un estudio minucioso tomando en cuenta la importancia de la cultura organizacional, lo que implica una adecuada gestión empresarial.

\section{Conclusiones}

Después de analizar la información se determinaron los beneficios de llevar una adecuada gestión administrativa, pues permite planear, organizar, dirigir y controlar una organización. Además, la estructuración de un organigrama ayuda a conocer las áreas, las funciones y las responsabilidades. Por su parte, una adecuada cultura organizacional, la implementación de manuales de organización, procedimientos y software administrativos ayudan a mejorar los procesos, a reducir gastos. Las MIPYMES en su formación y desarrollo se vuelven más sólidas cuando el líder es capaz de definir los propósitos a perseguir por la organización y las estrategias adecuadas para la realización de los procesos. El método empírico de administración ha pasado a la historia, por lo que el liderazgo actual está funcionando como pionero del crecimiento productivo y organizacional.

El capital humano es el elemento más importante de toda empresa, ya que es el único que puede administrar al resto de los recursos. Siempre se le debe motivar, capacitar e incentivar con los ingresos económicos que se merece para que la empresa pueda alcanzar sus objetivos. 


\section{Bibliografía}

Amaya, J. (2008). Manuales de Normas y Procedimientos. http://www.elprisma.com/ apuntes/administracion_de_empresas/manualesdenormasyprocedimientos/.

Chiavenato, I. (2006). Introducción a la Teoría General de la Administración. México: McGraw Hill.

Diario Oficial de la $\quad$ Federación (2009). Disponible http://www.dof.gob.mx/nota_detalle.php?codigo=5289919\&fecha=28/02/2013

Elizondo, M. M., Ríos, F. B., Cabrero, J. B., Morejón, V. M., Ramos, L. G. (2011). Análisis estratégico para el desarrollo de micro, pequeña y mediana empresa en el estado de Coahuila, México. Revista Internacional Administración y Finanzas (RIAF), 4(3), 1-19

ENAPROCE (2015). Disponible http://www.inegi.org.mx/est/contenidos/Proyectos/encuestas/establecimientos/otras/enaproce/def ault_t.aspx

Garibaldi, A., Rivera, S. (2015). Garibaldi Munguía, A., Rivera Rodríguez, S. (2015). El desarrollo de una ventaja competitiva a partir del capital humano en las empresas emergentes. (Spanish). Global Conference on Business \& Finance Proceedings, 10(1), 1100-1108.

Garibaldi, A., Rivera, S. (2015). Garibaldi Munguía, A., Rivera Rodríguez, S. (2015). El desarrollo de una ventaja competitiva a partir del capital humano en las empresas emergentes. (Spanish). Global Conference on Business \& Finance Proceedings, 10(1), 1100-1108.

Garza, C.R. (2000). Creación de PYMES: Objetivo emprendedor. Ingenierías, 3 (9), 54-58.

Garzón, J. (2012). Planeación Estratégica, Módulo XV, Maestría en Administración de Empresas, Universidad Técnica Estatal de Quevedo.

Hernández, R., Fernández, C. y Baptista, P. (2003). Metodología de la investigación. México: McGraw Hill. 
http://www.beta.inegi.org.mx/app/mapa/denue/. Consultado 21/11/2016 a las 14:00 horas.

INEGI (2015). Censos Económicos 2014. Resultados preliminares. México: INEGI.

Instituto Nacional de Estadística Geografía e Informática INEGI (2005).

Mochón, F. (2014). Administración, enfoque por competencias con casos Latinoamericanos. Editor S.A. de C.V México, primera edición.

PROMÉXICO. Disponible en: www.promexico.gob.mx

Saavedra, G. y Hernández, C. (2008). Caracterización e importancia de las MIPYMES en Latinoamérica. Actualidad Contable FACES. 11(17), 122-134.

Tapia, B. (2000). Apuntes de metodología de la investigación. México: Trillas.

Tunal, G. (2003). El problema de clasificación de las microempresas. Actualidad Contable FACES, 6 (7), 78-91.

Unger, K., Flores, D., Ibarra, J. E. (2014). Productividad y capital humano: Fuentes complementarias de la competitividad en los estados en México. Trimestre Económico, 81(4), 909-941.

Zevallos E. (2003). Micro, pequeñas y medianas empresas en América Latina. Revista CEPAL, 73, abril. 